\title{
Geschäftsmodelle im Internet der Dinge
}

\author{
Elgar Fleisch $\cdot$ Markus Weinberger · Felix Wortmann
}

Eingegangen: 31. August 2014 / Angenommen: 24. September 2014 / Online publiziert: 16. Oktober 2014 (C) Springer Fachmedien Wiesbaden 2014

Zusammenfassung Unternehmen, die heute primär in nicht-digitalen Branchen agieren, benötigen theoretisch und praktisch fundierte Hilfestellungen bei der Entwicklung und Umsetzung von Geschäftsmodellen im Internet der Dinge (Internet of Things, IoT). Durch unsere Untersuchung der Rolle des Internet in Geschäftsmodellen kommen wir zum Schluss, dass die Bedeutung des Internet in der Geschäftsmodellinnovation seit den 90er Jahren laufend zugenommen hat, dass jede Internet-Welle zu neuen digitalen Geschäftsmodellmustern geführt hat und dass die größten Umbrüche bisher in digitalen Branchen stattgefunden haben. Wir zeigen, dass digitale Geschäftsmodellmuster neu auch in der physischen Industrie relevant werden. Die Trennung von physischen und digitalen Branchen ist damit endgültig vorbei. Der Schlüssel dazu ist das IoT, das physische Produkte und digitale Services zu hybriden Lösungen verschmelzen lässt. Wir leiten eine sehr allgemein gehaltene Geschäftsmodelllogik für das IoT ab und stellen konkrete Bausteine und Muster

\footnotetext{
Ass. Prof. Dr. F. Wortmann $(\bowtie) \cdot$ Prof. Dr. E. Fleisch

Universität St. Gallen, Institut für Technologiemanagement, Dufourstrasse 40a, 9000 St. Gallen, Schweiz

E-Mail: felix.wortmann@unisg.ch

Prof. Dr. E. Fleisch

ETH Zürich, Department of Management, Technology and Economics, Weinbergstrasse 56/58, 8092 Zürich, Schweiz

Dr. M. Weinberger

Bosch Software Innovations, c/o Universität St. Gallen, Institut für Technologiemanagement, Dufourstrasse 40a, 9000 St. Gallen, Schweiz 
von Geschäftsmodellen vor. Für die zentralen Herausforderungen bei der Umsetzung solcher hybriden Geschäftsmodelle zeigen wir erste Lösungsansätze auf.

Schlüsselwörter Geschäftsmodelle · Geschäftsmodellmuster · Internet of Things $\cdot$ Cyber-Physical Systems

\section{Einfluss des Internet auf Geschäftsmodelle bis heute}

Ausgangspunkt der Überlegungen dieses Beitrags ist die Frage, wie und wo sich das Internet auf die Entwicklung von Geschäftsmodellen bislang ausgewirkt hat. ${ }^{1}$ Ihre Beantwortung ermöglicht einerseits einen schärferen Blick auf die Rolle der Informationstechnologie (IT) in der Geschäftsmodellinnovation bis heute, andererseits erlaubt sie einen qualifizierteren Ausblick auf mögliche weitere Geschäftsmodellinnovationen auf Basis neu entstehender Informationstechnologien, in diesem Fall auf Basis des Internet der Dinge (Internet of Things, IoT).

Als Grundlage dieser ersten Untersuchung dienen die Ergebnisse von Gassmann et al. (2013). Mehr als 300 Fallstudien zu Unternehmen werden hier analysiert, die die bisher gültige Logik in ihrer Branche durchbrochen und nachhaltig verändert haben. In jahrelanger Kleinarbeit haben Gassmann et al. diese Fallstudien nach Gemeinsamkeiten untersucht und ein Set von 55 sogenannten Geschäftsmodellmustern identifiziert. Dabei ist ein Geschäftsmodellmuster (Gassmann et al. 2013) „eine bestimmte Konfiguration der vier Kernelemente eines Geschäftsmodells (Wer sind die Kunden? Was wird verkauft? Wie stellt man es her? Wie realisiert man einen Ertrag?), welche sich in verschiedenen Firmen oder Industrien als erfolgreich erwiesen hat.“

\subsection{IT spielt eine zentrale Rolle in zahlreichen branchenverändernden Fallstudien und Geschäftsmodellmustern}

Wir haben nun untersucht, welchen Einfluss IT auf die 55 Geschäftsmodellmuster hat. Dabei unterscheiden wir drei verschiedene Rollen, welche IT in Geschäftsmodellmustern einnehmen kann:

- IT kann erstens konstituierend wirken, d. h. ohne sie kann ein Geschäftsmodellmuster nicht existieren. Beispiele sind die Geschäftsmodellmuster E-Commerce oder Crowdsourcing. Ohne IT sind sie nicht denkbar, deshalb bezeichnen wir sie als digitale Geschäftsmodellmuster.

- Zweitens kann IT aufwertend wirken. Muster wie Self Service haben auch vor der Ausbreitung von IT existiert und durch ihre Anwendung Brachen verändert. Mit IT, insbes. dem Internet, haben sie jedoch markant an Bedeutung im Sinne von Ausbreitung bzw. Marktanteil gewonnen.

- Drittens kann IT für ein Geschäftsmodellmuster irrelevant sein, wie etwa bei dem Geschäftsmodellmuster Franchising.

Bei einer detaillierten Analyse fällt auf, dass IT seit den 90er Jahren in sehr vielen Fallstudien eine hohe Bedeutung hat, auch wenn es nach wie vor branchenverän-

${ }^{1}$ Dieser Beitrag basiert im Wesentlichen auf dem Arbeitsbericht Fleisch et al. (2014). 
dernde Geschäftsmodellinnovationen gibt, die ohne IT auskommen. Dies ist einerseits nicht verwunderlich, denn IT wird erst seit den Neunzigern in der Breite in der Wirtschaft eingesetzt. Andererseits ist die Dichte der IT-getriebenen Fälle in den letzten Jahren beeindruckend. Ein Großteil der neueren Fallstudien beruht auf digitalen Geschäftsmodellmustern.

1.2 Jede neue Internet-Welle hat bisher zu neuen digitalen Geschäftsmodellmustern geführt

Trägt man die durch IT neu ermöglichten Geschäftsmodellmuster bzw. die mit dem Geschäftsmodellmuster assoziierten branchenverändernden Fallstudien auf der Zeitachse auf, so ergibt sich folgendes Bild (vgl. Abb. 1): Ein erstes Set an durch IT neu ermöglichten Geschäftsmodellmustern taucht zwischen 1995 und 2000 auf. Diese Geschäftsmodellmuster basieren alle auf dem sogenannten Web 1.0, als das Internet das erste Mal als Geschäftsinfrastruktur gesehen und verwendet wurde. Zu den neu ermöglichten Geschäftsmodellmustern laut Nomenklatur Gassmann et al. zählen z. B. E-Commerce oder auch Open Source (bezogen auf Software).

Um 2005 ist ein nächstes Set an IT-ermöglichten Geschäftsmodellmustern entstanden. Sie basieren allesamt auf dem Web 2.0, das Internet, das es auch ,einfachen“"Anwendern ermöglicht, beizutragen. Zu diesen Mustern zählen beispielsweise Crowdsourcing, Crowdfunding oder Long Tail.

1.3 Viele Internet-getriebene Geschäftsmodellmuster folgen drei übergeordneten Trends

Ob das IoT ebenfalls neue Geschäftsmodellmuster generiert - und falls ja, welche - ist die leitende Frage dieses Beitrags. Zu ihrer Beantwortung sind zwei weitere Erkenntnisse aus obiger Analyse hilfreich.

Zunächst folgen viele der IT-beeinflussten Geschäftsmodellmuster - unabhängig von der auslösenden Technologiewelle - drei übergeordneten Trends:

- Integration von Nutzern und Kunden: IT ermöglicht Unternehmen ein zunehmendes Einbinden ihrer Kunden in die Wertschöpfungskette. Mit anderen Worten: IT

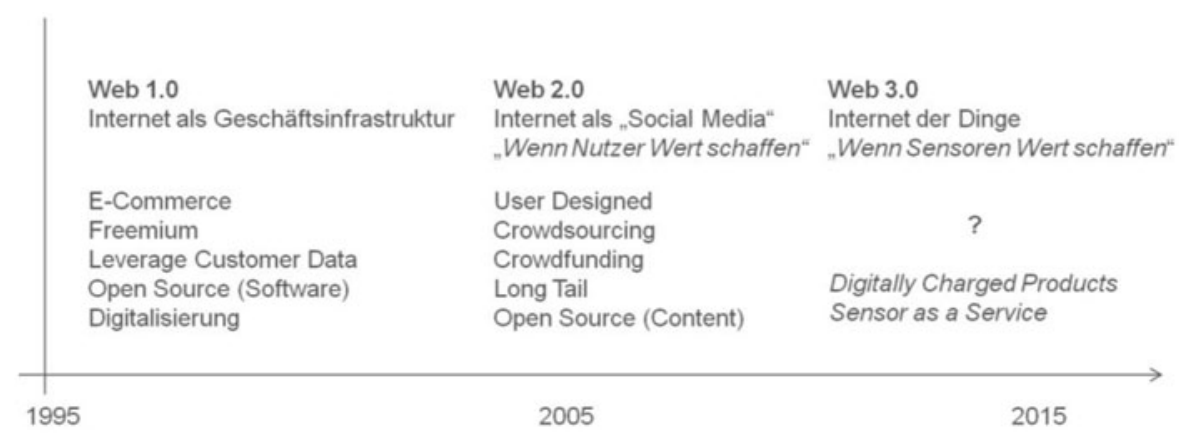

Abb. 1 Internet-Wellen und daraus neu entstandene digitale Geschäftsmodellmuster 
ermöglicht Unternehmen ihren Kunden Aufgaben zu übertragen. Beispiele liefern hier Geschäftsmodellmuster wie E-Commerce, Open Source (Content) oder Mass Customization.

- Dienstleistungsorientierung: Run Time Services bzw. der digitale Kontakt zum Kunden nach dem Verkauf nimmt zu. IT ermöglicht Unternehmen, die Kundenbeziehung auch nach dem Verkauf mittels IT-basierter Services aufrechtzuerhalten und zu nutzen. Beispielhafte Geschäftsmodellmuster hierzu sind Rent Instead of Buy, Subscription und Freemium.

- Kernkompetenz Analytics: Das zielgerichtete Sammeln und Analysieren von Transaktions- und Verwendungsdaten gewinnt an Bedeutung und ist eine Schlüsselfähigkeit für Produkt-, Preis- und Vertriebsgestaltung. Beispiele liefern hier die Geschäftsmodellmuster Subscription, Pay per Use und Performance-based Contracting.

1.4 Die großen Umbrüche brachten digitale Geschäftsmodellmuster bisher in digitalen Branchen

Die eindeutige Zuordnung der Rolle der IT zu den Geschäftsmodellmustern hat sich als herausfordernd erwiesen. Bei machen Geschäftsmodellmustern spielt die IT je nach Fallbeispiel eine unterschiedliche Rolle. Erst die Klassifikation der Fallbeispiele nach ihrer Zugehörigkeit zu digitalen bzw. nicht-digitale Branchen hat die notwendige Trennschärfe gebracht. Ein Unternehmen wird dabei einer digitalen Branche zugeordnet, wenn dessen Wesen digital ist.

Ein Beispiel hierzu liefert das Geschäftsmodellmuster Hidden Revenue (Unternehmen generiert Hauptumsatz nicht durch Produkte oder Dienstleistungen sondern durch Werbefläche, die daran geknüpft ist). JCDecaux hat schon 1964 ganz ohne IT mit seinen Stadtmöbeln, z. B. Bushaltestellen, den Werbemarkt verändert. In diesen nicht-digitalen Branchen wirkt IT auf das Geschäftsmodellmuster heute aufwertend. Für die Anwendung des Musters Hidden Revenue in Unternehmen wie Google oder Facebook, die digitalen Branchen zuzuordnen sind, wirkt IT jedoch zwingend konstituierend. IT hat nicht nur alte Geschäftsmodellmuster neu belebt und neue Geschäftsmodellmuster generiert, sie hat eine gesamte neue digitale Branche ermöglicht und in dieser Branche alte Geschäftsmodellmuster neu definiert.

Viele der digitalen Geschäftsmodellmuster wie z. B. Freemium (Produkt wird als freie Basis- und kostenpflichtige Premiumversion bereitgestellt) wurden bisher ausschließlich in der digitalen Welt angewendet. In der produzierenden Industrie hat das Internet bisher vor allem Abläufe vereinfacht und damit Kosten gespart sowie die Qualität und Variantenreichtum erhöht. Die großen Umbrüche hat das Internet in den digitalen Industrien gebracht wie Google, Facebook, Paypal, eBay, Youtube u. a. zeigen.

\section{Die betriebswirtschaftliche Kraft des IoT}

In der Entwicklung von Unternehmen wechseln sich Phasen evolutionärer, inkrementeller und revolutionärer, radikaler Veränderung ab (Rüegg-Stürm 2003). Auf 
Phasen kontinuierlicher Optimierung folgen immer wieder auch Phasen grundlegender Erneuerung. Dieser Abschnitt skizziert die gestalterische Kraft des IoT in der Wirtschaft. Zunächst steht dabei die Optimierung im Vordergrund. Anschließend wird aufgezeigt, warum und wie das IoT auch Ausgangspunkt für grundlegende Erneuerung sein kann. Eine breitere und fundiertere Analyse der ökonomischen Perspektive des IoT findet sich bei Fleisch et al. (2005) und Fleisch (2010).

\subsection{Das IoT ermöglicht High Resolution Management nun auch in der physischen Welt}

Die digitale Welt - und damit auch ihre Branchen - unterscheiden sich in zahlreichen Dimensionen von der physischen Welt und deren Branchen, z. B. in den Bereichen Grenzkosten in Produktion, Transport und Lagerhaltung, in Transport- und Produktionsgeschwindigkeit sowie in der Fähigkeit zur Abstraktion und Simulation.

Beispielsweise Google als prominenter Vertreter der digitalen Welt hat sich diese Eigenschaften zunutze gemacht und revolutioniert damit den Werbemarkt. Google analysiert Fragen in der Suchmaschine und Klicks auf Webseiten und misst so das Verhalten seiner Nutzer. Auf Basis dieser schärferen weil höher auflösenden Messdaten stellt die Firma jedem Nutzer zu jeder Zeit eine gewinnoptimale, individuelle Werbenachricht zu. Anhand der Nutzerreaktion misst Google wiederum in Echtzeit die Wirksamkeit des Banners, optimiert sein Allokationsmodell und verwendet dieselben Daten zur Rechnungsstellung an seine Werbekunden.

Die Auflösung des Regelkreises, den Google zum Management seiner digitalen Werbung verwendet, ist um ein vielfaches höher als in der physischen Welt, in der immer noch ein unidirektionales Medium wie Fernseher oder Plakatwand eine statische Botschaft an eine unbekannte Masse an potenziellen Kunden sendet. Eine feingranulare Steuerung bringt große Vorteile mit sich, daher entwickelt sich der digitale Werbemarkt wesentlich dynamischer als der physische. Die Werbebudgets fließen seit Jahren von der physischen in die digitale Welt ab.

Digitalisierung führt damit zu hochauflösendem Management (High Resolution Management), weil die Grenzkosten von Messung (in der Regelstrecke) und Aktuatorik (im Regler) gegen Null gehen und gleichzeitig Eingriffe nahezu in Lichtgeschwindigkeit stattfinden. Das IoT wendet diese Logik nun schrittweise auf die physische Welt an. Es steht für die Vision, in der jeder Gegenstand und Ort der physischen Welt Teil des Internet werden kann. Gegenstände und Orte erhalten dann meist einen Minicomputer und werden so zu smarten Dingen, die Informationen aus ihrer Umwelt aufnehmen und mit dem Internet bzw. anderen smarten Dingen kommunizieren können. Für den Menschen sind diese Minicomputer in der Regel kaum oder nicht sichtbar, der physische Teil des Gegenstands bleibt die wichtigste Schnittstelle für sie.

Smarte Dinge sind also Hybride, zusammengesetzt aus Teilen der physischen und digitalen Welt. Daher vereinen sie auch in der Anwendung die Gesetzmäßigkeiten beider Welten und führen so unter anderem das High Resolution Management in die physische Welt ein (Fleisch 2010). Folgendes Beispiel soll dies veranschaulichen: Weil die Messkosten in der physischen Lagerhaltung hoch sind, wird eine manuelle Messung so selten wie möglich durchgeführt - eine vollständige Inventur nur einmal 
pro Jahr. Der dazugehörende Managementregelkreis weist eine entsprechend niedrige Frequenz auf. Wenn nun IoT-Technologien vormals ,,agnostische“ Lagerbehälter und Regale ,smart“ machen, d. h. mit Sensorik und Kommunikationsfähigkeit ausstatten, dann können die smarten Behälter und Regale zu jederzeit zu Grenzkosten von Null ihren spezifischen Füllstand übermitteln. Weil Unternehmen nur managen können, was sie auch messen können, führen diese neuen Messfähigkeiten zu neuen Managementfähigkeiten. Im Fallbeispiel eines Produzenten von Schrauben haben diese einen neuartigen Nachfüll-Service hervorgebracht.

Das IoT wirkt auf die Betriebswirtschaftslehre ähnlich wie das Ultraschallgerät auf die Medizin oder das Rasterelektronenmikroskop (REM) auf die Physik. Mit den Technologien des IoT lassen sich Dinge vermessen und erkennen, die vorher nicht (wirtschaftlich) erkennbar waren. Ultraschall und REM trieben jeweils ihre gesamte Disziplin voran.

\subsection{Digitale Geschäftsmodellmuster werden neu auch in der physischen Industrie relevant}

Soll nun der Befestigungstechnikproduzent mit seinem ,smarten“ Regal die Bestandsinformationen seinen Kunden gratis zur Verfügung stellen? Oder als einen mit der physischen Lieferung integrierten Bezahlservice von Anfang an? Und: Wem gehören die Daten? Dem Kunden, in dessen Hallen sie entstehen, oder dem Lieferanten; ihm gehören ja die Behälter, die die Daten generieren? Können und sollen die Daten - anonymisiert, quer über die gesamte Kundenbasis - wertvolle und zeitnahe Entwicklungen in der Branche zeigen und im Rahmen des Geschäftsmodellmusters Leverage Customer Data kapitalisiert werden?

Wie dem auch sei, Leverage Customer Data ist ein Beispiel dafür, dass plötzlich Geschäftsmodellmuster, die bisher den digitalen Branchen vorbehalten waren, auch für die klassischen, physischen Branchen relevant werden. Vielmehr noch, im IoT vermengen sich zwangsweise digitale Geschäftsmodellmuster mit solchen aus der nicht-digitalen Welt zu einem hybriden Konstrukt, wie aus den Wertschöpfungsstufen einer abstrakten IoT-Anwendung besonders gut sichtbar wird.

Die Wertschöpfungsstufen einer IoT-Anwendung (vgl. Abb. 2) sind Ergebnis einer Analyse zahlreicher Anwendungen, die heute in Wissenschaft und Praxis dem IoT zugeordnet werden. Folgender Abschnitt erklärt sie am Beispiel einer „smarten“ LED-Lampe.

Ebene 1 - Physisches Ding: Der physische Teil einer Lösung, in diesem Fall die LED-Lampe, bildet die erste Ebene des Wertschöpfungsmodells. Sie liefert den ersten direkten und physischen Nutzen an den Anwender - in Form von Wohlbehagen durch Licht. Weil die Lampe physischer Natur ist, ist sie immer an einen Ort gebunden und kann ihren Nutzen auf dieser Ebene nur in ihrer direkten Umgebung, beispielsweise in einem Raum liefern. Die Geschäftsmodellmuster für den Verkauf oder die Vermietung von LED-Lampen sind den LED-Lampenproduzenten hinlänglich bekannt.

Ebene 2 - Sensor/Aktuator: Ebene 2 fügt dem physischen Ding eine Minicomputer mit Sensorik und Aktuatorik hinzu. Die Sensorik misst lokale Daten, der Aktuator liefert lokale Services und erzeugt damit lokalen Nutzen. Im Beispiel der LED-Lampe 


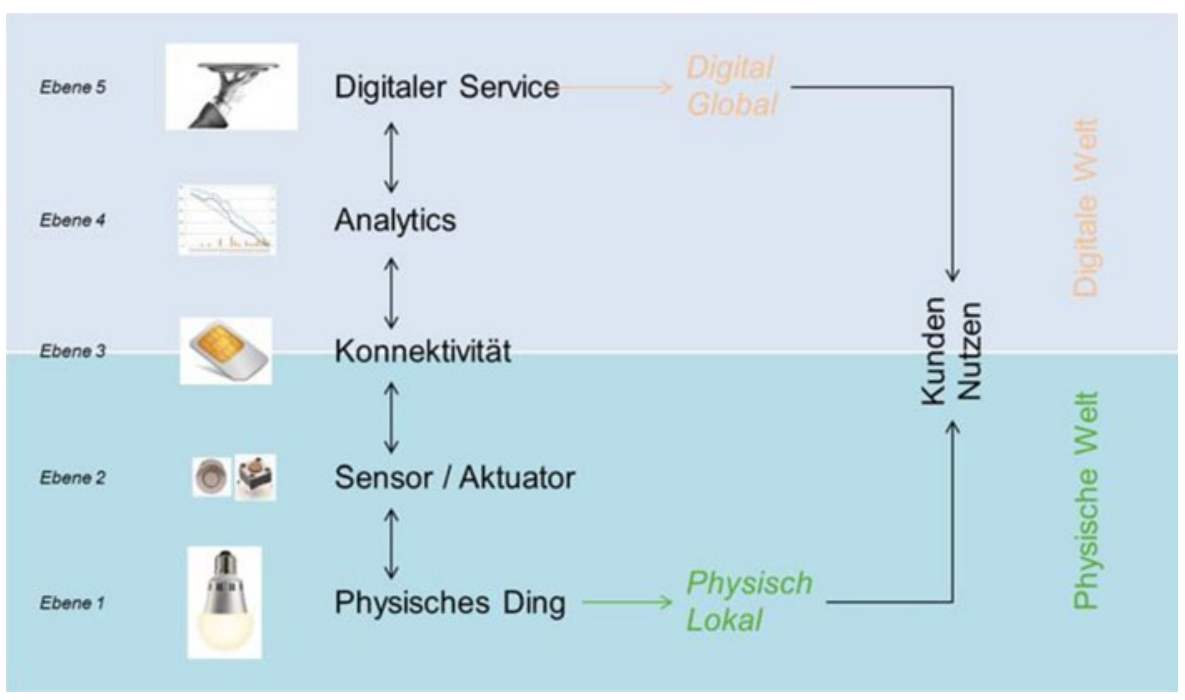

Abb. 2 Wertschöpfungsstufen einer Anwendung im IoT

misst ein Anwesenheitssensor laufend recht zuverlässig und kostengünstig, ob Menschen im Raum präsent sind. Der Aktuator schaltet die Lampe in Abhängigkeit der Anwesenheit automatisch ein und aus und liefert damit lokalen Nutzen - auch, weil die ,smarte“ LED-Lampe ohne separaten, verkabelten Bewegungsmelder auskommt und somit Anwesenheit per se erkennen kann.

Ebene 3 - Konnektivität: Mit der Ebene 3 erhalten die unteren Ebenen, insbesondere Sensoren und Aktuatoren, einen Zugang zum Internet und damit globalen Zugriff. Die Lampe aus unserem Beispiel wird über ein eingebautes Funkmodul adressierbar und kann ihren Zustand autorisierten Abonnenten auf der ganzen Welt zu vernachlässigbaren Grenzkosten bekanntgeben.

Eben 4 - Analytics: Konnektivität per se liefert keinen Mehrwert. Ebene 4 sammelt, speichert, plausibilisiert und klassifiziert Sensordaten, webt Erkenntnisse anderer Webservices mit ein und errechnet Konsequenzen für die Aktuatorik - typischerweise in einem Cloud-basierten Backendsystem. Im LED-Beispiel speichert Ebene 4 u. a. die Ein- und Ausschaltzeiten von Lampen in einem Haushalt, klassifiziert Bewegungsmuster und führt die Betriebsstunden einzelner Lampen mit.

Ebene 5 - Digitaler Service: Auf dieser obersten Ebene werden die Möglichkeiten aus den unteren Ebenen in digitale Dienstleistungen strukturiert, in geeigneter Form verpackt - beispielsweise als Webservice oder mobile Applikation - und global zur Verfügung gestellt. Für diese digitalen Dienstleistungen, die untrennbar mit den Datengenerierenden ,smarten“ Dingen verbunden sind, gelten die Eigenschaften digitaler Geschäftsmodellmuster.

Aus der LED-Lampe mit Anwesenheitssensor wird erst hier eine Sicherheitslampe, die auf Wunsch bzw. App-Knopfdruck ihres Besitzers Anwesenheit vorspielt, im Fall eines unwillkommenen Eindringlings einen Alarm an den Besitzer, seine Nachbarn oder die Polizei absetzt, oder im „Fight-Back-Modus“ den Einbrecher mit 
rotem Blitzlicht zu vertreiben versucht - und das alles wiederum zu vernachlässigbaren Grenzkosten.

Eine wichtige Erkenntnis ist, dass die Ebenen 1 bis 5 nicht unabhängig voneinander erstellt werden können. Daher sind die verbindenden Pfeile bidirektional gezeichnet. Eine werthaltige IoT-Lösung ist i.d. R nicht die reine Addition der Ebenen, sondern eine bis in die physische Ebene hineinreichende Integration. Der Bau der Hardware wird damit zunehmend von den darüber liegenden digitalen Ebenen beeinflusst. Eine getrennte Betrachtung der Ebenen wird viele attraktive digitale Services nicht ermöglichen können. Die Verschränkung von Hardware- und Internetlösungsentwicklung erscheint immer mehr als zwingende Notwendigkeit.

\subsection{Physische Produkte und digitale Services verschmelzen zu hybriden Lösungen}

Auf einer sehr abstrakten Ebene kann die Logik von Geschäftsmodellen im IoT auf eine einfache Formel reduziert werden (vgl. Abb. 3).

Sie besagt, dass der Wert einer IoT-Lösung auf der Herstellerseite aus der Kombination eines klassischen, in der Vergangenheit nicht mit dem Internet verknüpften Produktes besteht, das mit IT, genauer mit den Ebenen 2 bis 4 aus obigem Modell, veredelt wird. Dieser Wert entfaltet sich auf der Kundenseite auf Ebene 5 als Nutzen aus dem physischen Produkt und den damit verbundenen digitalen Services. Dabei entsteht ein Ganzes, das mehr ist als die Summe der Ebenen, die auf dem Produkt aufbauen - insbesondere wegen der einfachen und wenig kostenintensiven Kombinierbarkeit von eigenen und externen digitalen Services.

Eine Uhr von Limmex (www.limmex.com) ist auch noch eine Uhr, wenn sie über GSM-Modul, Mikrofon, Lautsprecher und eigene Homepage verfügt. Ihr physischer und lokaler Nutzen ist immer noch - neben der Angabe der Uhrzeit - das Signalisieren von Eigenschaften ihres Trägers über ihr schickes, zeitloses Design an Dritte

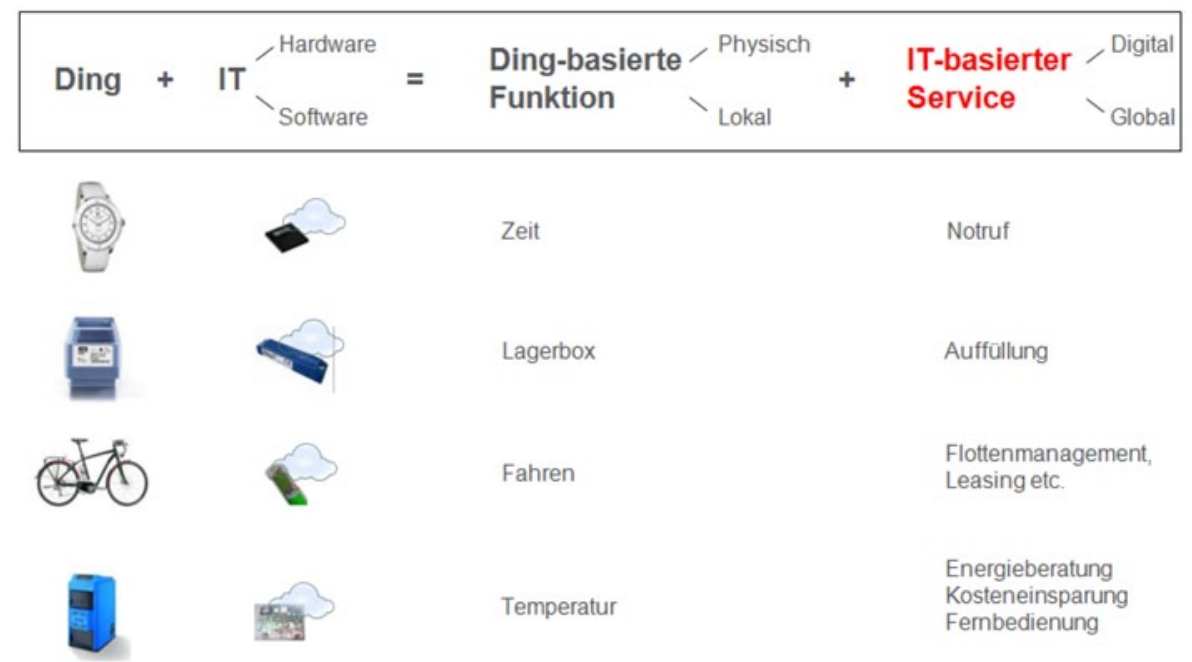

Abb. 3 Die Produkt-Service-Logik des IoT 
am Kaffeetisch. Zusätzlich wird sie zum Notruf an Familie, Freunde oder das Rote Kreuz, den der Träger im Internet selber konfigurieren kann. Die „smarte“ mit einem Long Range RFID-Chip aufgeladene Lagerbox von Intellion (www.intellion.com) ist immer noch eine Lagerbox und bietet Platz für Schrauben und Beilagscheiben. Zusätzlich ermöglicht sie einen neuen wettbewerbsdifferenzierenden Nachfüllservice für den Schraubenlieferanten. Die Liste an hybriden Produkt-Service-Angeboten von Startups, etablierten Unternehmen und Ideen aus der Forschung wächst jeden Tag. Das Startup qipp (www.qipp.com) beispielsweise treibt diese Logik ins Extreme und liefert eine Infrastruktur, mit deren Hilfe jedes Unternehmen seine physischen Produkte sehr einfach und kostengünstig mit standardisierten, global verfügbaren, digitalen Services aller Couleur aufladen kann.

\section{Geschäftsmodellmuster im IoT}

Das anwendungsorientierte Ziel dieses Beitrags ist die Ableitung von theoretisch und praktisch fundierten Hilfestellungen für die Entwicklung von Geschäftsmodellen im IoT. Diese sollen in erster Linie inspirieren, so abstrakt sein, dass sie branchenübergreifend anwendbar sind und gleichzeitig so konkret, dass sie für Innovatoren aus Wirtschaft und Gesellschaft handlungsleitend wirken.

Dazu haben wir die 55 Geschäftsmodellmuster von Gassmann et al. (2013) sowie zahlreiche IoT-Anwendungen in den Dimensionen Wertschöpfungsstufen und High Resolution Management analysiert. In letzterer sind die Möglichkeiten und Limitation der technischen Fähigkeiten des IoT enthalten. Das Ergebnis dieser Analyse lässt sich über sechs Bausteine für Geschäftsmodellmuster und zwei eigenständige Geschäftsmodellmuster im IoT darstellen. Im Folgenden beschreiben wir lediglich Bausteine und Muster, die das IoT neu ermöglicht. Nicht diskutiert werden können hier die 20 Muster, die von den aufgezeigten Bausteinen begünstigt werden.

\subsection{Bausteine, die bestehende Geschäftsmodellmuster digital erweitern}

Physical Freemium steht für ein physisches Gut, das inklusive eines kostenfreien digitalen Service verkauft wird, beispielsweise einer digitalen Montage-, Betriebs- und Wartungsanleitung, die gratis am Produkt „,klebt“. Ein Teil der Kunden entscheidet sich im Laufe der Zeit für darüber hinausgehende Premium Services, die verrechnet werden, beispielsweise eine elektronische Fernüberwachung oder ein Benchmarking über die gesamte Kundenbasis hinweg. Das New Yorker Startup Canary (www. canary.is) bietet etwa eine Smart Home-Alarmanlage an, die verschiedene Sensoren, von Temperatur- oder Bewegungssensoren bis zu einer Kamera enthält. Die Grundfunktion, einen Raum während der Abwesenheit des Bewohners zu überwachen und bei Unregelmäßigkeiten eine Nachricht an eine Smartphone App zu schicken, ist im Preis des Geräts enthalten. Darüber hinaus wurden während der CrowdfundingKampagne auf der Plattform Kickstarter weitere kostenpflichtige Dienstleistungen angekündigt, z. B. zusätzlicher Speicherplatz für aufgezeichnete Vorgänge oder die Nutzung eines Call Centers. Ein vergleichbares Paket bietet sehr viel erfolgreicher 
das Unternehmen Dropcam (www.dropcam.com) an, das im Juni 2014 fünf Jahre nach seiner Gründung für 555 Mio. USD von Google Nest gekauft wurde.

Digital Add-on bezeichnet einen Geschäftsmodellbaustein, in dem ein physisches Gut sehr preisgünstig, d. h. mit geringer Marge, verkauft wird. Im Lauf der Zeit kann der Kunde zahlreiche margenstarke digitale Services dazu erwerben bzw. freischalten lassen. Wenn die Leistung eines Autos per Software konfiguriert werden kann und das Fahrzeug ein Knoten im Internet ist, dann kann sich der Kunde beispielsweise für das kommende Wochenende 50 PS dazukaufen. Und wenn Add-on Services auch von Dritten angeboten werden, dann kann sich der Kunde sehr einfach eine passende, zusätzliche Mikroversicherung für die Ausfahrt in Italien beschaffen. Die Verkaufsprovision geht an den Hersteller des Autos oder einen Dritten.

Die erfolgreiche Anwendung der Geschäftsmodellmuster Razor and Blade und Lock-in setzt durch, dass nur Originalkomponenten mit einem System kompatibel sind. Beispielsweise können nur Gillette Rasierklingen mit Gillette Rasierern verwendet werden. In vielen Fällen werden Wettbewerber durch Patente daran gehindert, kompatible Komponenten in ein solches System zu liefern. Digital Lock-in in physischen Produkten steht für einen Sensor-basierten, digitalen „Handshake“, der u. a. zur Einschränkung der Kompatibilität, Verhinderung von Fälschungen und Sicherstellung von Garantieleistungen eingesetzt wird.

\subsection{Bausteine, die die Anwendbarkeit bestehender Geschäftsmodellmuster für physische Dinge erweitern}

Physische Produkte werden durch Product as Point of Sales zum Träger digitaler Verkaufs- und Marketingservices, die der Kunde direkt am Gegenstand oder mittelbar via Smartphone und Identifikationstechnologie konsumiert. Die Kaugummipackung wird zum e-Shop, jeder Gegenstand kann Träger digitaler Werbung sein, das Produkt sammelt und kommuniziert Loyalty-Punkte selbständig und fächert seine Erlebniswelt digital über Smartphones auf. Die Erweiterung von Dingen zu Verkaufsstellen ist in manchen Beispielen bereits Realität. Richtet man die Kamera eines Smartphone auf ein Produkt, öffnet sich ein Internetshop, der den Kauf desselben Produkts, von Ersatzteilen, Zubehör, Verbrauchsmaterial oder zugehörigen Dienstleistungen anbietet. Die „Amazon App“ bietet als ein Beispiel diese Funktion bereits heute für Produkte, die einen Barcode tragen und im Sortiment von Amazon enthalten sind.

Der Baustein Object Self Service bezeichnet die Möglichkeit, dass Dinge autonom Bestellungen im Internet auslösen. Ein Heizsystem könnte beispielsweise Öl nachbestellen, sobald ein bestimmter Füllstand im Öltank unterschritten wird. Die Idee des Self Service ist also nicht mehr auf den Kunden beschränkt, auch Dinge können sich selbstbedienen. Im Sinne des Geschäftsmodellmusters Direct Selling werden dabei Intermediäre umgangen. Solution Provider Geschäftsmodelle werden durch den automatischen Nachbezug von Verbrauchsmaterial vereinfacht.

„Smarte“ Dinge können Daten über ihren eigenen Zustand oder den ihrer Umwelt in Echtzeit übertragen. Dadurch werden (präventive) Fehlerentdeckung sowie die Überwachung der Nutzung und beispielsweise der Füllstände von Verbrauchsmaterial möglich (Remote Usage and Condition Monitoring). Bisher war die dafür erforderliche Technologie kompliziert und relativ teuer. Mit fortschreitender Verbrei- 
tung des IoT verringern sich die Kosten und der erforderliche Aufwand, wodurch die Anwendung dieser Technologie auch bei geringerwertigen Gütern rentabel wird. Der Computerzubehör-Hersteller Brother bietet beispielsweise Leasingverträge für Laserdrucker ohne Basisleasingrate an - nur die tatsächlich gedruckten Seiten werden abgerechnet. In diesem Beispiel wird also das Pay per Use-Geschäftsmodellmuster auf Produkte im Wert von nur wenigen hundert Euro angewendet. Die technische Grundlage für eine effiziente Umsetzung des Geschäftsmodells liegt in der Übertragung der relevanten Daten an den Anbieter über das Internet.

\subsection{Eigenständige Geschäftsmodellmuster im IoT}

Die oben genannten Bausteine sind alle Spielarten der Idee, dass das IoT in seinen Anwendungen jeweils physische Produkte mit digitalen Dienstleistungen zu einem hybriden Bündel aus einem Guss verschränkt. Dabei können die Services einfacher oder komplexerer Natur sein, sie können vom Hersteller des Produktes oder von Dritten angeboten werden, sie können nahe am Produkt sein oder in ihrer Vernetzung bei Vierten eine völlig andere Bedeutung erlangen. Der Begriff Digitally Charged Products bildet die Klammer um die zusammengehörenden Bausteine. Aufgrund der Mächtigkeit der dahinterliegenden Ideen, gehen wir davon aus, dass sich Digitally Charged Products als neues Geschäftsmodellmuster etabliert: Klassische physische Produkte werden mit neuen Sensor-basierten digitalen Dienstleistungsbündel aufgeladen und mit neuem Wertversprechen positioniert. Die Beispiele hierzu sind die bereits erwähnte Sicherheitslösung an der LED-Lampe, die e-Kanban Lösung an der Kiste, der Notruf an der Uhr. Mit Digitally Charged Products erfahren bekannte Service-orientierte Geschäftsmodellmuster eine neue Relevanz in physischen Industrien.

Auch die Idee, dass Sensordaten eines Gewerks gesammelt, aufbereitet und gegen Entgelt anderen Gewerken zur Verfügung gestellt werden, hat große Mächtigkeit. Deshalb schlagen wir sie unter dem Begriff Sensor as a Service als Geschäftsmodellmuster des IoT vor. Die Messwerte aus der physischen Welt werden dabei nicht mehr vertikal integriert, nur für genau eine Anwendung erhoben, gespeichert und aufbereitet, sondern vielmehr für eine breite Palette von potenziellen Anwendungen - für ein Ökosystem, dessen Entstehung im IoT sicherlich eine der nächsten großen Herausforderungen darstellt (Schuermans und Vakulenko (2014). Anders als bei Digitally Charged Products stehen hier nicht mehr die datengenerierenden Produkte oder die resultierenden Dienstleistungen im Mittelpunkt, sondern die Daten selber. Sie sind die primäre Währung, die es zu bewirtschaften gilt. Die Firma Streetline (www. streetline.com) liefert hierzu ein gutes Beispiel. Sie installiert in Städten und auf privaten Grundstücken Sensoren, die die Belegung von Parkplätzen erkennen können mit dem Zweck, die erhobenen Daten an interessierte Dritte zu verkaufen. Die Autofahrer erhalten die Informationen über eine App heute gratis. Für die Behörden sind die etwas anders aufbereiteten Daten von hohem Wert: Ihr physischer Aufwand, um Parksünder zu identifizieren, sinkt dramatisch, die Auslastung der Parkplätze steigt, die Informationen zur Optimierung ihrer Infrastruktur gewinnen an Qualität. Sensor as a Service steht für ein Geschäftsmodellmuster, in dessen Zentrum sich ein ,,multisided“ Markt für Sensordaten befindet. 


\section{Unternehmerische Herausforderungen bei der Umsetzung von Geschäftsmodellen im IoT}

In der Zusammenarbeit mit zahlreichen Unternehmen, von Großkonzernen bis hin zu Startups zeigte sich - für erfahrene Führungskräfte wenig überraschend -, dass die Ideengenerierung, der sich der Schwerpunkt dieses Beitrags widmet, die kleinere der Hürden bei einer Etablierung eines neuen Geschäftsmodells im IoT darstellt. Im Folgenden gehen wir kurz auf die zentralen Herausforderungen bei der Umsetzung ein, die insbesondere bei Unternehmen auftreten, die eine erfolgreiche Historie im klassischen Geschäft mit physischen Produkten haben: Die produzierende Industrie bzw. das produzierende Gewerbe.

\subsection{Produkt- versus Servicegeschäft}

Zur Frage nach dem optimalen Mix von Produkt- und Servicegeschäft hat sich in den letzten zehn Jahren in Wirtschaft und Wissenschaft ein breiter Diskurs entwickelt, den die Verschmelzung von der physischen mit der digitalen Welt neu belebt, denn der digitale Teil einer hybriden Lösung ist immer eine Dienstleistung. Die zentralen Fragestellungen, die in der Literatur und von Unternehmen bearbeitet werden, lauten (Fischer et al. 2012): Wie viel und welches Servicegeschäft ist angemessen? Gibt es Entwicklungsstufen auf dem Pfad einer produktdominanten zu einer servicedominanten Organisation? Wie kann die Dienstleistungsentwicklung, -vermarktung und -erbringung optimal organisiert werden - auf regionaler wie auf internationaler Ebene? Welche Dienstleistungskategorien gibt es? Wie überzeuge ich Kunden für ehemals kostenlose Dienstleistungen zu bezahlen? Wie sieht die Preisfindung aus? Wie organisiere und incentiviere ich meine Verkaufsorganisation?

Dienstleistungen unterscheiden sich grundsätzlich von Produkten. Sie sind beispielsweise nicht lagerbar, werden in der Regel beim Kunden in Zusammenarbeit mit ihm erbracht und häufig mit mehreren kleinen, zeitlich verteilten Beträgen entgolten. Im Kern geht es darum, die strategischen und operativen Eigenschaften von Produkten und Dienstleistungen gegeneinander abzuwägen und in einem nachhaltig optimalen Verhältnis zu halten.

Die Besonderheit im IoT ist, dass der Serviceanteil in den hier skizzierten Geschäftsmodellen immer digitaler Natur ist. Dies hat zwei Konsequenzen: Erstens, muss die Theorie und Praxis der Serviceorientierung vor dem Hintergrund der Eigenschaften digitaler Dienstleitungen kritisch hinterfragt und allenfalls erweitert werden. Zweitens führt eine in das Produkt hineinreichende Digitalisierung (im Gegensatz zur digitalen Unterstützung von Wertschöpfungsprozessen) zwangsweise zu einer weiteren Dienstleistungsorientierung.

\subsection{Zusammenprall der Hardware- und Internetkultur}

Die unterschiedlichen Eigenschaften von physischen und digitalen Produkten machen sich insbesondere in der Produktentwicklung bemerkbar. Wenn die Grenzkosten einer Produktmodifikation gering sind, ist die Entwicklung dann gut organisiert, wenn der Managementregelkreis angemessen kurz und hochfrequent ist. In der 
digitalen Welt, insbesondere im Internet, sind demzufolge agile Entwicklungsprozesse heute Standard. Nahezu jedes erfolgreiche Internetsoftwareunternehmen und -projekt verwendet heute die Methode SCRUM und testet jeden Abend ein neues sichtbares Ergebnis, um es den Kunden zur Verwendung zu übergeben. In einer Welt, in der ein „Bug“ mittels eines nahezu kostenfreien Updates selbst bei einer Installed Base in Millionenhöhe ohne weiteres repariert werden kann, und in der es oft genug aufgrund der Netzwerkeffekte von Beginn an um möglichst hohe Wachstumszahlen geht, zählen in der Entwicklung vor allem Geschwindigkeit, früher Kundenkontakt und Ästhetik. Die Stichworte lauten hier „Minimum Viable Product“ (Ries 2009) - eine Produktversion, die bei minimalem Aufwand maximale Erkenntnisse über den Kunden liefert - und Perpetual Beta (O’Reilly 2005) - die Auflösung ,fertiger““ Releases zu Gunsten kontinuierlicher Weiterentwicklung des Produktes.

Im Hardwaregeschäft aber auch in der Welt des Embedded Computing gelten andere Randbedingungen. Hier führt beispielsweise ein Fehler in einem bereits verkauften Produkt in der Regel zu höchst kostspieligen und imageschädigenden Rückrufaktionen. Diese technisch-ökonomisch bedingten Unterschiede haben zu divergenten Kulturen in Hardware- und Internetsoftwareabteilungen geführt und vermeintlich inkompatible Organisationseinheiten geformt.

Das technische Delta lässt sich nicht wegdefinieren. Jedoch lässt sich das Wissen über das jeweils andere Fachgebiet bis zur Anschlussfähigkeit aufbauen. Dies sorgt bei den Schlüsselmitarbeitern für die notwendige Offenheit für gewinnbringenden Austausch und die Bereitschaft Best Practices aus dem anderen Lager zu übernehmen.

Jedes Atom, das mit wirtschaftlichem Vorteil durch ein Bit abgelöst werden kann, wird aus den weiter oben genannten Gründen auch abgelöst. Die Digitalisierung von Hardwarefunktionen nimmt zu. Damit gewinnt auch die Frage an Bedeutung und Brisanz, wer in der Entwicklung hybrider Lösungen die „Oberleitung“ innehat, die Hardware- oder die Softwareseite? Die richtige Antwort hängt sicher vom Serviceanteil im vorliegenden Geschäftsmodell ab. Ohne quantitative empirische Untersuchung lässt sich nur eine Aussage machen, die auf lückenhaftes anekdotisches Wissen zurückgreift: Immer öfter obsiegt hier die Softwareseite.

Viele der untersuchten IoT-Lösungen weisen heute Eigenschaften von disruptiven Innovationen (Christensen 1997) auf. Sie positionieren sich mit einem völlig neuen Werteversprechen, sind damit kaum vergleichbar und adressieren einen neuen Markt. Sie sind klein, relativ kostengünstig und - mit herkömmlichen Metriken gemessen - qualitativ minderwertig und margenschwach. Die ex ante Erstellung eines Business Case ist häufig mit großen Unsicherheiten verbunden. Daher liegt es nahe, die Lösungsvorschläge, die Christensen Unternehmen zur Bewältigung von disruptiven Innovationen anbietet, auch bei der Entwicklung hybrider IoT-Produkte zu testen. Der Schlüssel liegt dort in der Schaffung oder der Übernahme von kleinen, selbständigen unternehmerischen Einheiten, die in ihrer Dynamik, Gehalts- und Reportingstruktur der Größe ihres Zielmarktes entsprechen und die unabhängig von bisherigen Kunden und Kapitalgebern agieren können. 


\subsection{Umgang mit Anwendungsdaten}

Hybride Lösungen bedeuten in den meisten Fällen, dass der Anbieter Zugriff auf Daten haben muss, die permanent aus der Anwendung der Lösung entstehen. Für klassische, produzierende Unternehmen ist dies neu und birgt zahlreiche Chancen aber auch Risiken.

$\mathrm{Zu}$ den Chancen zählen datenbasierter und feingranularer, unverfälschter und lückenloser Input für die Weiterentwicklung der Lösung bzw. für die Entwicklung neuer Angebote, für die Optimierung von Kundensegmentierung, Ansprache, Ertragsmodell und Preisfindung und für die dynamische, situationsspezifische, automatische Konfiguration des Angebots während der Laufzeit. Der professionelle Umgang mit diesen Massendaten, heute unter den Begriffen Analytics, Big Data oder Data Science diskutiert, ist eine neue grundlegende Fähigkeit, die Unternehmen besitzen oder aufbauen müssen, um diese Chancen zu nutzen. Aus diesem Grund haben O'Reilly (2005) und Andere markant festgestellt: „SQL is the new HTML“ bzw. „Data Science is cool".

$\mathrm{Zu}$ den Herausforderungen zählen sämtliche Fragen rund um die informationelle Selbstbestimmung der Anwender, insbesondere jene zur bestimmungsgerechten Verwendung sowie zur Sicherheit der Daten. Wem gehören die aus der Anwendung generierten Daten? Dem Anwender, dem Lösungsanbieter, beiden? Der relativ junge Ansatz, den u. a. Pentland (2009) verfolgt, erscheint hier vielversprechend. Er sieht Daten als Gut, das dem Erzeuger der Daten gehört. Dieser kann frei entscheiden, was er mit dem Gut machen möchte. Er kann es dabei wie Geld behandeln und nach Gutdünken behalten, spenden oder gegen eine andere Währung oder eine Gegenleistungen verkaufen. Fest steht, dass jede hybride Lösung eine klare und für allen Seiten transparente und sicher implementierte Vorstellung braucht, wie sie mit Anwendungsdaten umgeht, die beim Kunden generiert wurden. Nur so kann, für Kunden wie Anbieter nachhaltig, Nutzen aus diesen Daten gezogen werden.

\section{Einige Antworten und viele offene Fragen}

Der vorliegende Beitrag verfolgt das Ziel, Innovatoren aus Wirtschaft und Gesellschaft zu Geschäftsmodellen im IoT zu inspirieren. Er analysiert dazu die Rolle, die das Internet in Geschäftsmodellen bis heute einnimmt, dokumentiert die spezifische ökonomische Energie des IoT und leitet daraus eine generelle Produkt-Service Logik ab, die als Grundlage für konkrete Bausteine und Muster von Geschäftsmodellen im IoT dienen. Abschließend zeigt er einige Schlüsselherausforderungen bei deren Umsetzung auf, mit denen insbesondere Unternehmen mit einer erfolgreichen Geschichte in der produzierenden Industrie konfrontiert sind.

Zahlreiche Aspekte, die in direktem Zusammenhang mit IoT-Geschäftsmodellen stehen, beleuchtet der Artikel nicht. Beispielsweise klammert er die aktuell sehr prominent geführte Diskussion rund um technische Standards auf den unterschiedlichsten Ebenen der Kommunikation insbesondere in der letzten Meile (vom „smarten“ Ding zum ersten klassischen Internetcomputer) aus, ebenso die rasante Evolution drahtloser Protokolle, die alles bestimmende Energiefrage, Fragen der Systemro- 
bustheit, -wartbarkeit und -sicherheit. Er verzichtet außerdem auf die Darstellung der Topographie und der Entwicklung des Anbietermarktes, die spezifischen Rollen, die Hersteller entlang der Ebenen in Abb. 2 haben. Auch kann der Beitrag nicht auf branchen- oder prozessspezifische Anwendungen eingehen. Die zentrale Rolle des Mobiltelefons, das im IoT als Medium zwischen Menschen, „smarten“ Dingen bzw. ,smarter“ Umgebung und dem Internet vermittelt wird ebenso wenig behandelt, wie die Emotionalisierung der physischen Welt. Diese tritt dann ein, wenn Dinge in Echtzeit auf ihre Umgebung reagieren, zu einem wenigstens gefühlten Leben erwachen (Kelly 1999) und das Verhalten der Umgebung inklusive der Menschen in einer neuen Qualität beeinflussen.

Der vorliegende Beitrag wirft mehr offene Fragen auf, als er beantwortet. Einige können nun jedoch konkreter gefasst werden. Heute ist es aufschlussreich zu lesen, was vor zehn Jahren zum IoT geschrieben wurde. In 10 Jahren wird es interessant sein, zurückzublicken und zu sehen, welche der hier aufgezeigten Entwicklungen und Begriffe sich als nachhaltig erwiesen haben und welche in den Hintergrund getreten sind. Akademisch und wirtschaftlich bleibt das IoT ein faszinierendes und lohnendes Phänomen.

Danksagung Dieser Beitrag entstand im Rahmen des Bosch Internet of Things Lab der Universität St. Gallen.

\section{Literatur}

Christensen C (1997) The innovator's dilemma: when new technologies cause great firms to fail. Harvard Business Review Press, Cambridge

Fischer T, Gebauer H, Fleisch E (2012) Service business development: strategies for value creation in manufacturing firms. Cambridge University Press, Cambridge

Fleisch E (2010) What is the internet of things? An economic perspective. Auto-ID Labs White Paper WPBIZAPP-053. ETH Zürich \& University of St. Gallen, St. Gallen

Fleisch E, Christ O, Dierkes M (2005) Die betriebswirtschaftliche Vision des Internets der Dinge. In: Fleisch E, Mattern F (Hrsg) Das Internet der Dinge. Springer, Berlin, S 3-37

Fleisch E, Weinberger M, Wortmann F (2014) Geschäftsmodelle im Internet der Dinge. Arbeitsbericht. http://www.iot-lab.ch/wp-content/uploads/2014/09/GM-im-IOT_Bosch-Lab-White-Paper.pdf. Gesehen 1. Oktober 2014

Gassmann O, Frankenberger K, Csik M (2013) Geschäftsmodelle entwickeln: 55 innovative Konzepte mit dem St. Galler Business Model Navigator. Hanser Verlag, München

von Hippel E (1986) Lead users. A source of novel product concepts. Manag Sci 32:791-805

Kelly K (1999) New rules for the new economy: 10 radical strategies for a connected world. Penguin Books, New York

O'Reilly T (2005) What Is Web 2.0. http://oreilly.com/web2/archive/what-is-web-20.html. Zugegriffen: 29. Aug 2014

Pentland A (2009) Reality mining of mobile communications: toward a new deal on data. In: Dutta S, Mia I (Hrsg) The Global Information Technology Report 2008-2009, World Economic Forum. S 75-80

Ries E (2009) Minimum viable product: a guide. http://www.startuplessonslearned.com/2009/08/minimum-viable-product-guide.html. Zugegriffen: 29 Aug 2014

Rüegg-Stürm J (2003) Das neue St. Galler Management-Modell. Haupt, Bern

Schuermans S, Vakulenko M (2014) IOT - breaking free from internet and things, how communities and data will shape the future of IoT in way's we can't imagine. VisionMobile Report 